\title{
Hypertrophic pachymeningitis associated with Sjögren's syndrome: case report and literature review
}

\author{
Chihiro Kondo ${ }^{1}$, Takayasu Mishima ${ }^{1}$, Hidekazu Mera ${ }^{1}$, Shinsuke Fujioka ${ }^{1}$, Hironori Fukumoto ${ }^{2}$, \\ Tooru Inoue $^{2}$, Katsuhisa Miyake ${ }^{3}$, Mikiko Aoki ${ }^{4}$, Kazuki Nabeshima ${ }^{4}$, Yoshio Tsuboi ${ }^{1}$ \\ ${ }^{1}$ Department of Neurology, Faculty of Medicine, Fukuoka University, Fukuoka, Japan \\ ${ }^{2}$ Department of Neurosurgery, Faculty of Medicine, Fukuoka University, Fukuoka Japan \\ ${ }^{3}$ Division of Nephrology and Rheumatology, Department of Internal Medicine, \\ Faculty of Medicine, Fukuoka University, Fukuoka Japan \\ ${ }^{4}$ Department of Pathology, Faculty of Medicine, Fukuoka University, Fukuoka, Japan
}

\section{To the Editors}

A 60-year-old woman presented with fever and headache. Neurological examination was unremarkable. However, anti-SS-A (Sjögren's Syndrome-A) and anti-SS-B antibodies were detected in the serum without dry eye or dry mouth. Later, generalised tonic convulsions occurred and administration of levetiracetam was initiated. She was referred to our department due to the presence of dysesthesia on the left side of the body. Neurological examination confirmed dysesthesia in the left side of the body including the face. Deep tendon reflex was exaggerated in all extremities, with positive Hoffmann and Trömner's reflexes. Meningeal irritation signs were not observed. Serum immunological tests, including those for angiotensin converting enzyme (ACE), proteinase-3-anti-neutrophil cytoplasmic antibodies (PR3-ANCA), and myeloperoxidase-anti-neutrophil cytoplasmic antibodies (MPO-ANCA), were negative. Serum IgG4 level was within the normal range. Saxon and Schirmer's tests were positive, and the patient was diagnosed with Sjögren's syndrome (SS). Cerebrospinal fluid (CSF) examination showed a slight elevation of protein level with a normal glucose level and cell count. Brain magnetic resonance imaging (MRI) showed thickened dura mater in the right parietal region in a diffusion-weighted image (DWI) and in a fluid-attenuated inversion recovery image (FLAIR) (Fig. $1 \mathrm{~A}$ and $\mathrm{B})$. Hypertrophic pachymeningitis (HP) was suspected, and a dural biopsy was performed.

The pathological findings showed mild fibrosis and infiltration of inflammatory cells. No malignant lesion or presence of vasculitis was detected (Fig. 1C and D). Immunohistochemically, cerebral cortex on the surface staining revealed both
$\mathrm{CD} 3$ and $\mathrm{CD} 20$ positive lymphocytes, and CD68-positive microglia, in the perivascular region. All of these glial cells were negative for IDH-1, and ATRX expression was well retained. Although dural pathology showed only mild inflammation, due to the exclusion of other diseases we diagnosed HP associated with SS. After the dural biopsy, the patient was treated with two courses of intravenous methylprednisolone $(1 \mathrm{~g} /$ day) for three days. Soon after the treatment, the patient's headache and dysesthesia improved, and oral prednisolone $(50 \mathrm{mg} /$ day) was started, followed by gradual tapering. A brain MRI 94 days after the treatment showed an improvement of the thickened region (Fig. 1E and F). Currently, the patient's treatment comprises oral prednisolone at $10 \mathrm{mg}$ /day without the recurrence of symptoms.

$\mathrm{HP}$ is a rare disease characterised by thickened dura mater. This disease often presents with headache, fever, visual loss, and double vision with accompanying cranial nerve pathology (frequently in cranial nerves II-VIII). Occasionally, HP occurs idiopathically. Infection, sarcoidosis, and certain autoimmune diseases have been reported as secondary causes of HP. In a nationwide survey of $159 \mathrm{HP}$ patients conducted in Japan, the underlying disease was revealed to be idiopathic in $44 \%$, ANCA-related in $30.2 \%$, and IgG4/multifocal fibrosclerotic systemic disease-related in $8.8 \%$ of the cases [1]. In our review of the literature, nine cases of HP associated with SS have been reported worldwide (Table S1). Consistent with the presented case, frequent clinical features in these nine cases were fever and headache that developed following neurological symptoms such as increased deep tendon reflexes, sensory disorders, or muscle weakness. The site of dural thickening was variable: three cases of spinal thickening and seven cases in intracranial

Address for correspondence: Yoshio Tsuboi, Department of Neurology, Fukuoka University, 7-45-1, Nanakuma, Johnan-ku, Fukuoka 814-0180, Japan; tel: +81-92-801-1011 (ext. 3520), e-mail: tsuboi@cis.fukuoka-u.ac.jp

Received: 17.08.2021 Accepted: 28.12.2021 Early publication date: 31.01 .2022

This article is available in open access under Creative Common Attribution-Non-Commercial-No Derivatives 4.0 International (CC BY-NC-ND 4.0) license, allowing to download articles and share them with others as long as they credit the authors and the publisher, but without permission to change them in any way or use them commercially. 


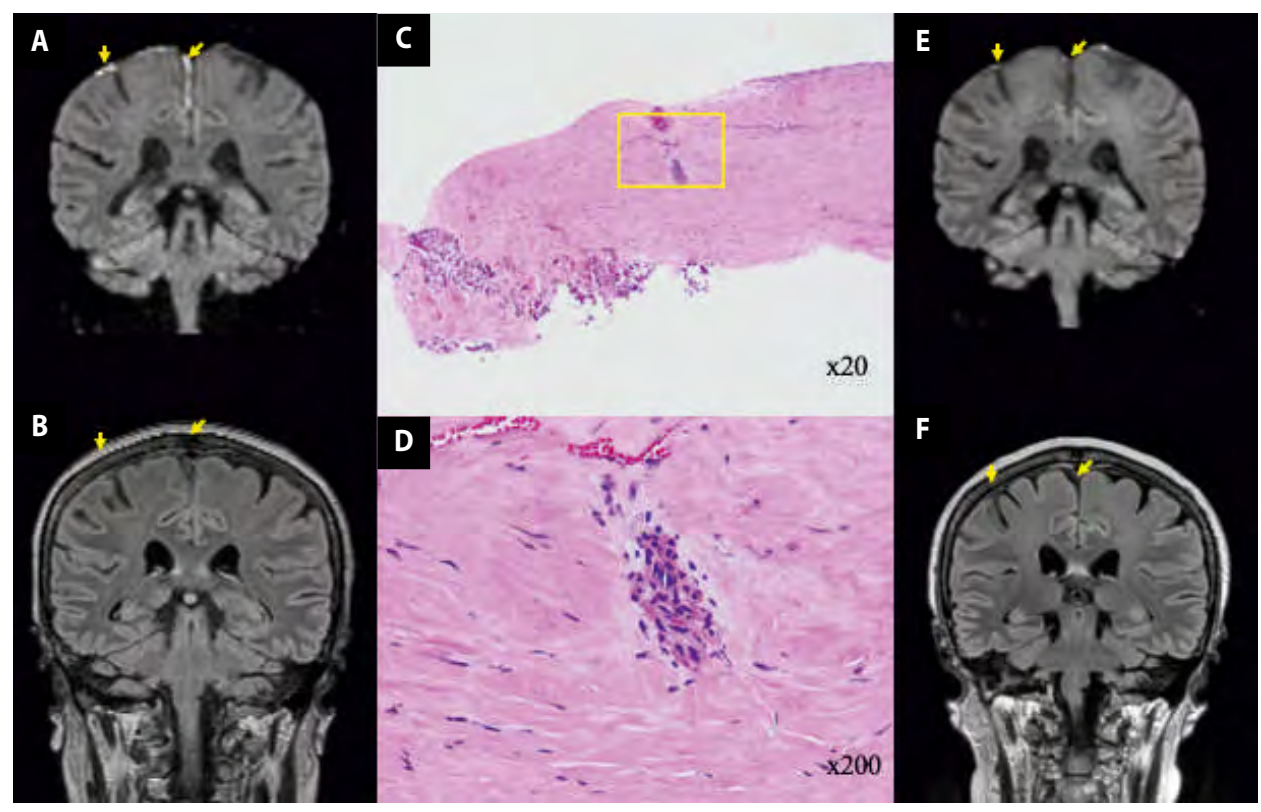

Figure 1. Magnetic resonance imaging (MRI) of brain with thickened dura mater at right parietal region in diffusion-weighted (DWI); A. Fluidattenuated inversion recovery image (FLAIR); B. Follow-up MRI did not show dura mater thickening (E, F). Photomicrographs of sections of dura mater tissue stained with hematoxylin and eosin showed thickened dura mater (magnification 20x); C. Mild fibrosis and infiltration of inflammatory cells (magnification 200x) (D)

dural thickening. Regarding pathological findings, a dural biopsy was also performed in 52 of the HP patients included in the Japanese survey. The findings primarily revealed fibrosis and infiltration of inflammatory cells, with some cases showing granuloma. IgG4-positive plasma cells were observed in 11 cases (seven cases of IgG4/multifocal fibrosclerotic systemic disease-related and four ANCA-related cases) [1]. In ANCA-related HP, the pathological findings mainly show fibrosis and infiltration of inflammatory cells, and sometimes IgG4 positive cells or granulomas [2-4]. In HP associated with SS, dural biopsy has been performed in five cases, including the present case, revealing nonspecifically fibrosis and infiltration of inflammatory cells, which may be the pathological characteristics of this condition.

Regarding treatment, idiopathic or autoimmunity-associated HP is usually treated with steroid therapy as the firstline treatment. In some cases of idiopathic HP, and those of ANCA- and IgG4-related HP, additional immunosuppressant treatment may be required. The present case was successfully treated with steroid therapy without recurrence. Other reported cases of SS-associated HP were also treated with steroid therapy alone, and eight of the nine cases showed no recurrence. Only one case was treated with an immunosuppressant. Nakano et al. reported that moderate-dose steroids may be sufficient for the treatment of HP in SS (Table S1). In addition to hypertrophic pachymeningitis, aseptic meningitis is another central nervous system disorder associated with Sjogren's syndrome. Contrast-enhanced MRI is necessary to distinguish between meningitis and HP, and a dural biopsy should be performed if possible.

In conclusion, our case report and review of the literature reveal that the pathological findings of HP associated with SS show nonspecific regions. In addition, we conclude that steroid therapy should be started immediately after the diagnosis of HP associated with SS because such therapy is confirmed to be effective.

Conflict of interest: None. Funding: None.

\section{References}

1. Yonekawa T, Murai H, Utsuki S, et al. A nationwide survey of hypertrophic pachymeningitis in Japan. J Neurol Neurosurg Psychiatry. 2014; 85(7): 732-739, doi: 10.1136/jnnp-2013-306410, indexed in Pubmed: 24273222.

2. Matsumoto K, Akiyama M, Kajio N, et al. Adolescent PR3-ANCA-positive hypertrophic pachymeningitis: A case report and review of the literature. Medicine (Baltimore). 2018; 97(17): e0521, doi: 10.1097/ MD.0000000000010521, indexed in Pubmed: 29703022.

3. Chen $\mathrm{H}$, Zhang $\mathrm{W}$, Jing J, et al. The clinical and imaging features of hypertrophic pachymeningitis: a clinical analysis on 22 patients. Neurol Sci. 2019; 40(2): 269-274, doi: 10.1007/s10072-018-3619-4, indexed in Pubmed: 30377845.

4. Bi Z, Shang Ke, Cao J, et al. Hypertrophic pachymeningitis in Chinese patients: presentation, radiological findings, and clinical course. Biomed Res Int. 2020; 2020: 2926419, doi: 10.1155/2020/2926419, indexed in Pubmed: 32879880. 